\title{
Muscle-specific integrins in masseter muscle fibers of chimpanzees: an immunohistochemical study
}

\author{
Angelo Favaloro', Giampiero Speranza', Silvia Rezza ${ }^{2}$, Valentina Gatta ${ }^{3}$, \\ Gianluigi Vaccarino ${ }^{1}$, Liborio Stuppia ${ }^{3}$, Felice Festa ${ }^{2}$, Giuseppe Anastasi ${ }^{1}$
}

\author{
${ }^{1}$ Department of Biomorphology and Biotechnologies, University of Messina, Italy \\ ${ }^{2}$ Department of Odontostomatologic Science; ${ }^{3}$ Department of Biomedical Science G. \\ D'Annunzio University Foundation, Chieti, Italy
}

\begin{abstract}
Most notably, recent comparative genomic analyses strongly indicate that the marked differences between modern human and chimpanzees are likely due more to changes in gene regulation than to modifications of the genes. The most peculiar aspect of hominoid karyotypes is that human have 46 chromosomes whereas gorillas and chimpanzees have 48. Interestingly, human and chimpanzees do share identical inversions on chromosome 7 and 9 that are not evident in the gorilla karyotype. Thus, the general phylogeny suggests that humans and chimpanzees are sister taxa; based on this, it seems that human-chimpanzee sequence similarity is an astonishing $99 \%$. At this purpose, of particular interest is the inactivation of the myosin heavy chain 16 (MYH16) gene, most prominently expressed in the masticatory muscle of mammals. It has been showed that the loss of this gene in humans may have resulted in smaller masticatory muscle and consequential changes to cranio-facial morphology and expansion of the human brain case. Powerful masticatory muscles are found in most primates; contrarily, in both modern and fossil member Homo, these muscles are considerably smaller. The evolving hominid masticatory apparatus shifted towards a pattern of gracilization nearly simultaneously with accelerated encephalization in early Homo. To better comprehend the real role of the MYH16 gene, we studied the primary proteins present in the muscle fibers of humans and non-humans, in order to understand if they really can be influenced by MYH16 gene. At this aim we examined the muscle-specific integrins, $\alpha 7 \mathrm{~B}$ and $\beta 1 \mathrm{D}$-integrins, and their relative fetal isoforms, $\alpha 7 \mathrm{~A}$ and $\beta 1 \mathrm{~A}$-integrins, analyzing, by immunohistochemistry, muscle biopsies of two components of a chimpanzee's group in captivity, an alpha male and a non-alpha male subjects; all these integrins participate in vital biological processes such as maintenance of tissue integrity, embryonic development, cell differentiation, and cell-extracellular matrix interactions. Our results demonstrated a different quantitative composition of integrins, in alpha male in respect to human and non-alpha male, hypothesizing that the MYH16 gene could modify the expression of integrins, influencing, in turn, the phenotype of muscle. In this way, $\alpha 7 \mathrm{~A}-$ and $\beta 1 \mathrm{~A}$-integrin could determine the presence of type II fibers and then they could play a key role in the determination of contraction force. Then, MYH16 gene could be a common interactor of signalling between sarcoglycans and integrins in chimpanzee muscles.
\end{abstract}

Key words: skeletal muscle, chimpanzee, integrin, sarcoglycan, immunohistochemistry

\section{Introduction}

The ancient researches of Huxley [1] and Darwin [2] affirmed that the modern humans and extant chimpanzees share striking similarities. However, in full century later, the molecular methods were used to examine this relationship. Despite the readily apparent physical similarities shared by African apes to the

Correspondence: A. Favaloro, Dipartimento di Biomorfologia

e Biotecnologie, Policlinico Universitario, Torre Biologica,

Via Consolare Valeria, 1, IT-98125 - Messina, Italy;

e-mail: angelo.favaloro@unime.it exclusion of modern humans (body hair, knucle-walking, thin tooth enamel), the molecular support for a human-chimpanzee clade is now overwhelming [3].

Most notably, recent comparative genomic analyses strongly indicate that the marked differences between modern human and chimpanzees are likely due more to changes in gene regulation than to modifications of the genes [4]. The most peculiar aspect of hominoid karyotypes is that human have 46 chromosomes whereas gorillas and chimpanzees have 48 [5]. Human chromosome 2 seems to be a fusion of ape chromosomes 12 and 13. However, other Authors speculated 
that this fusion may be very recent and perhaps even accounts for the supposed lack of interbreeding between modern humans and Neanderthals [6].

Interestingly, human and chimpanzees do share identical inversions on chromosome 7 and 9 that are not evident in the gorilla karyotype [7]. Thus, the general phylogeny suggests that humans and chimpanzees are sister taxa; based on this, it seems that humanchimpanzee sequence similarity is an astonishing $99 \%$ [8]. Furthermore, a small genetic change could have a dramatic phenotypic impact if it modified the expression patterns of other genes. These regulatory changes are now being identified, including some likely to underlie cognitive differences between chimpanzees and human $[4,9]$.

At this purpose, of particular interest is the inactivation of the myosin heavy chain 16 (MYH16) gene, most prominently expressed in the masticatory muscle of mammals [10]. It has been showed that the loss of this gene in humans may have resulted in smaller masticatory muscle and consequential changes to craniofacial morphology and expansion of the human brain case [10]. In fact, experimental models of masticatory muscle resection or transposition have demonstrated the correlation between cranio-facial morphology and the force of masticatory muscle contraction $[11,12]$.

Besides, powerful masticatory muscles are found in most primates; contrarily, in both modern and fossil member Homo, these muscles are considerably smaller [13]. The evolving hominid masticatory apparatus shifted towards a pattern of gracilization nearly simultaneously with accelerated encephalization in early Homo [14].

The differences between modern humans and chimpanzees are most evident also in gross anatomical comparison which showed that the relative size of individual masticatory muscle homologues contrast remarkably; for example, there are evident differences in the sites of attachment of the masseter and temporal muscles in chimpanzees and human skulls [10]. At the histological level, the difference between human and non-human primate temporalis muscle is demonstrated by staining for type II sarcomeric myosin and interstitial laminin.

To better comprehend the real role of the MYH16 gene, we studied the primary proteins present in the muscle fibers of humans and non-humans, in order to understand if they really can be influenced by MYH16 gene. At this aim we examined the muscle-specific integrins, $\alpha 7 \mathrm{~B}$ and $\beta 1 \mathrm{D}$-integrins, and their relative fetal isoforms, $\alpha 7 \mathrm{~A}$ and $\beta 1 \mathrm{~A}$-integrins.

Integrins are a family of transmembrane receptor molecules, which participate in vital biological processes such as maintenance of tissue integrity, embryonic development, cell differentiation, and cellextracellular matrix interactions [15-18]. These pro- teins also have an important role in transducing mechanical and chemical signals into the cells [17-19]. They are heterodimers composed of two subunits, $\alpha$ and $\beta$, both containing extracellular, transmembrane, and cytoplasmic domains [20].

In particular, the $\beta$ subunits are responsible of interaction with the cytoskeleton, whereas a subunits participate in the determination of the specificity of the ligand binding and signalling. Previously, it has been demonstrated that the $\beta 1 \mathrm{D}$ integrin displaces the $\beta 1 \mathrm{~A}$ isoform in striated muscle [21].

About $\alpha 7$ subunit, the $\alpha 7 \mathrm{~A}$ and $\alpha 7 \mathrm{C}$ isoforms appear to be restricted to skeletal muscle, in contrast to $\alpha 7 \mathrm{~B}$, which is also expressed in nonmuscle cells. In skeletal muscle, $\alpha 7 \mathrm{~B}$ is expressed in proliferating, mobile myogenic precursor cells and it is still detected in adult myofibers with restricted localization to neuromuscular junctions and myotendinous junctions [22]. Thus $\alpha 7 \mathrm{~A}$ may have a specific role in regenerating muscle during the dynamic adhesion stage, whereas in mature skeletal muscle it appears to have a minor role [20].

Other investigations have demonstrated that the enhanced expression of the $\alpha 7 \beta 1$ reduces muscular dystrophy and restore viability in dystrophic mice [22]. On this, recently we examined muscle fibers during muscular inactivity, showing that the fetal isoforms of muscle-specific integrins can be displace the adult isoform due to loss of regulatory effects on gene expression of these protein which could provoke a quantitative modification of transmembrane receptors $[23,24]$.

Besides it was demonstrated a bidirectional signalling between integrins and sarcoglycans [25-28]. This bidirectional signalling, by an common interactor as filamin2, could permit the transduction of mechanical and chemical signalling into the cells and the maintenance of the cell integrity.

On this basis, here we analyzed for first time, the $\alpha 7 \mathrm{~A}-, \alpha 7 \mathrm{~B}-\beta 1 \mathrm{~A}-$, and $\beta 1 \mathrm{D}-i n t e g r i n$, by immunohistochemical technique, analyzing muscle biopsies of two components of a chimpanzee's group in captivity, an alpha male and a non-alpha male subjects.

\section{Materials and methods}

Tissue samples. Initially, we examined human skeletal muscle biopsies of masseter from two patients who did not have any coexisting neuromuscular pathology, but had undergone orthopaedic surgery for other reasons.

The age of the patients was between 30 and 60 years and all gave their informed consent. The investigation conformed with the principles outlined in the Declaration of Helsinki.

Subsequently, we examined masseter muscle biopsies from two adult males (32 and 30 years old) chimpanzees (Pan troglodytes). They were part of a larger social group (7 animals) living in a indoor/outdoor enclosure at the "Zoosafari di Fasano" (BR, Italy). 
Muscle biopsies are done using anesthesia and therefore require prior immobilization of the chimpanzee with a chemical agent. The drug to chimpanzees are administered through a process known as "a knockdown" in which pre-loaded syringes are fired through a dart gun. After administration of anesthesia and immobilization of animals, the biopsy are effectuated with excision of cute, subcutaneous tissues and muscle fascia; finally, we used a 6.0 $\mathrm{mm}$ biopsy punch. The biopsy specimens were analyzed using immunohistochemistry technique.

Immunohistochemistry. The biopsies were fixed in $3 \%$ paraformaldehyde in $0.2 \mathrm{M}$ phosphate buffer, $\mathrm{pH} 7.4$, for 2 hours at room temperature. They were then washed extensively with 0.2 $\mathrm{M}$ phosphate buffer, $\mathrm{pH} 7.4$, and then with phosphate-buffered saline (PBS), containing $12 \%$ and $18 \%$ sucrose. The samples were snap-frozen in liquid nitrogen and $20 \mu \mathrm{m}$ sections were prepared in a cryostat for their use in a protocol to perform immunofluorescence. The sections were placed on glass slides that were coated with $0.5 \%$ gelatin and $0.005 \%$ chromium potassium sulphate.

To block non-specific binding sites and to permeabilize the membranes, the sections were preincubated with $1 \%$ bovine serum albumin (BSA), $0.3 \%$ Triton X-100 in PBS at room temperature for $15 \mathrm{~min}$. Finally, the sections were incubated with primary antibodies. The following primary antibodies were used: anti- $\alpha$-sarcoglycan diluted 1:100, anti- $\beta$-sarcoglycan diluted $1: 200$, anti- $\gamma$-sarcoglycan diluted $1: 100$, anti- $\delta$-sarcoglycan diluted 1:50, and anti- $\varepsilon$-sarcoglycan diluted 1:100 (all from Novocastra Laboratories); anti- $\alpha 7 \mathrm{~B}$-integrin diluted 1:50, anti- $\beta 1 \mathrm{D}$-integrin diluted 1:50, anti- $\alpha 7 \mathrm{~A}$-integrin diluted 1:100, and anti- $\beta 1 \mathrm{~A}-$ integrin diluted 1:50 (synthetic peptides from the $\mathrm{COOH}$ terminal region; kindly provided by the laboratory of Professor Tarone, University of Torino). Primary antibodies were detected using Texas Red-conjugated IgG (Jackson ImmunoResearch Laboratories, Inc.). Slides were finally washed in PBS and sealed with mounting medium.

The sections were then analyzed and images acquired using a Zeiss LSM 5 DUO confocal laser scanning microscope. All images were digitalized at a resolution of 8 bits into an array of $2048 \times 2048$ pixels. Optical sections of fluorescent specimens were obtained using a HeNe laser (wavelength $=543 \mathrm{~nm}$ ) and an Argon laser (wavelength $=458 \mathrm{~nm}$ ) at a 1-min 2-sec scanning speed with up to 8 averages; $1.50 \mu \mathrm{m}$-thick sections were obtained using a pinhole of 250 . For each reaction, at least 100 individual fibres were examined. Contrast and brightness were established by examining the most brightly labelled pixels and choosing the settings that allowed clear visualization of the structural details while keeping the pixel intensity at its highest ( 200). Each image was acquired within $62 \mathrm{sec}$, in order to minimize photodegradation.

Digital images were cropped and figure montages prepared using Adobe Photoshop 7.0 (Adobe Systems; Palo Alto, CA).

\section{Results}

In this report we obtained and studied $20 \mu$ m-thick cryosections by acquiring a stack of 16 sections, with a scan step size of $0.8 \mu \mathrm{m}$.

Firstly, we analyzed the samples of the human subject, performing immunofluorescence reactions using a single antibody each time. Our results, on longitudinal sections, showed normal intermittent, or periodic, staining patterns for all tested integrins. Moreover, immunostaining of $\alpha 7 \mathrm{~A}-$ (Fig. 1A), and $\beta 1 \mathrm{~A}$-integrin (Fig. 1B) was reduced compared to that of $\alpha 7 \mathrm{~B}$ - (Fig. $1 \mathrm{C}$ ), and $\beta 1 \mathrm{D}$-integrin (Fig. 1D). About sarcoglycans, it was showed a normal staining pattern of all these tested proteins (Fig. 2).

Secondly, we performed immunostaining on longitudinal sections of masseter muscle fibers from a nonalpha male chimpazee. All of the tested integrin isoforms showed the same behaviour of human samples. In particular, $\alpha 7 \mathrm{~A}-$ (Fig. 3A), and $\beta 1 \mathrm{~A}$-integrin (Fig. 3B) showed normal periodic and reduced staining patterns, whereas immunostaining of $\alpha 7 \mathrm{~B}$ - (Fig. 3C), and $\beta 1 \mathrm{D}$-integrin (Fig. 3D), showed a normal fluorescence. Also sarcoglycan immunostainings showed the same situation observed in human muscle (Fig. 4).

Finally, we tested the same integrin isoforms in alpha male chimpanzee. Our observations showed the same normal intermittent staining patterns for all tested proteins, but interestingly immunostaining of $\alpha 7 \mathrm{~A}$ (Fig. 5A), and $\beta 1 \mathrm{~A}$-integrin (Fig. 5B) was clearly increased compared to that of $\alpha 7 \mathrm{~B}-$ (Fig. $5 \mathrm{C}$ ), and $\beta 1 D$-integrin (Fig. 5D) which showed a reduced but detectable fluorescence, and to that of $\alpha 7 \mathrm{~A}$ - and $\beta 1 \mathrm{~A}$ integrin of human and non-alpha male chimpanzee. The sarcoglycan subcomplex analysis in the alpha male showed a normal fluorescence of all tested sarcoglycans as well as in the human and non-alpha male muscle fibers (Fig. 6).

\section{Discussion and conclusion}

In this study we demonstrated evident differences of protein arrangement between muscle fibers of human and chimpanzees. We also showed clear differences between protein arrangement between muscle fibers of alpha male and non-alpha male chimpanzees of the same group. In particular, by immunofluorescence technique, in human and in non-alpha male we highlighted a clear staining patterns for $\alpha 7 \mathrm{~B}$ and $\beta 1 \mathrm{D}$ integrin, whereas $\alpha 7 \mathrm{~A}$ - and $\beta 1 \mathrm{~A}$-integrin showed a weak staining pattern. Interestingly, in muscle fibers of alpha male we observed an opposite situation showing a very strong fluorescence for $\alpha 7 \mathrm{~A}$ and $\beta 1 \mathrm{~A}$-integrin, and a weak staining pattern for $\alpha 7 \mathrm{~B}$ and $\beta 1 D$-integrin. There are no evident differences in all tested sarcoglycans.

The integrins transmit signals by organizing the cytoskeleton, thus regulating cell shape and internal cellular architecture. In fact, integrin cytoplasmic domains interact with cytoskeletal components $[29,30]$. In this view, appropriate cell shape and cytoskeletal organization can regulate the biosynthetic capabilities of the cell, and thus contribute to cell growth or differentiation $[31,33]$. Furthermore, integrins are deemed to be true receptors capable of giving rise to biochemical signals within the cell [18]. Previously it is demonstrated that high levels of $\alpha 7 \mathrm{~A}$ were detected in differentiating myotubes, but in proliferating myoblast only the $\alpha 7 \mathrm{~B}$ isoform was present 

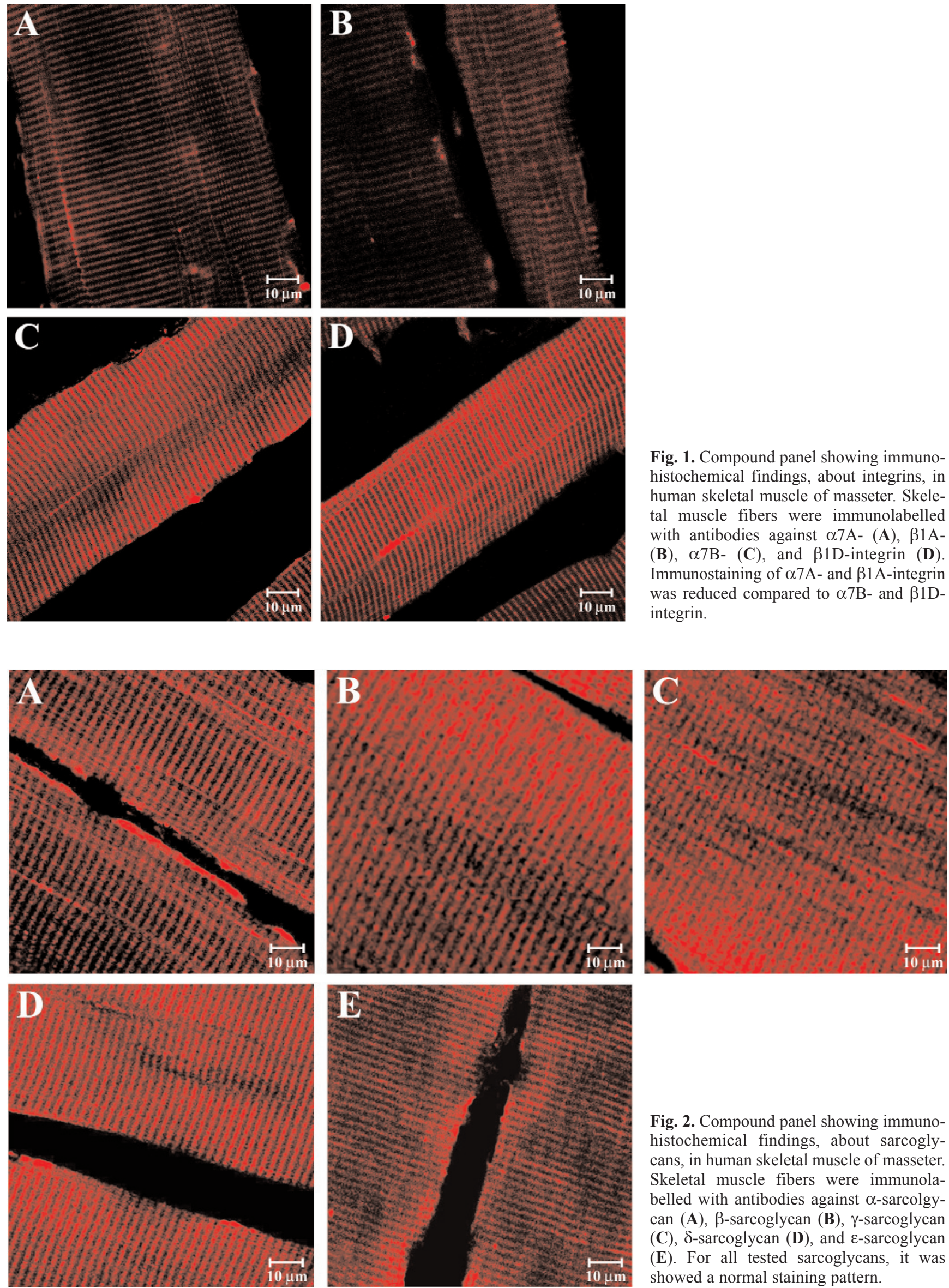

Fig. 2. Compound panel showing immunohistochemical findings, about sarcoglycans, in human skeletal muscle of masseter. Skeletal muscle fibers were immunolabelled with antibodies against $\alpha$-sarcolgycan (A), $\beta$-sarcoglycan (B), $\gamma$-sarcoglycan (C), $\delta$-sarcoglycan (D), and $\varepsilon$-sarcoglycan (E). For all tested sarcoglycans, it was showed a normal staining pattern.
Fig. 1. Compound panel showing immunohistochemical findings, about integrins, in tal muscle fibers were immunolabelled with antibodies against $\alpha 7 \mathrm{~A}-(\mathbf{A}), \beta 1 \mathrm{~A}$ (B), $\alpha 7 \mathrm{~B}-(\mathbf{C})$, and $\beta 1 \mathrm{D}$-integrin (D). Immunostaining of $\alpha 7 \mathrm{~A}$ - and $\beta 1 \mathrm{~A}$-integrin was reduced compared to $\alpha 7 \mathrm{~B}$ - and $\beta 1 \mathrm{D}$ integrin. 

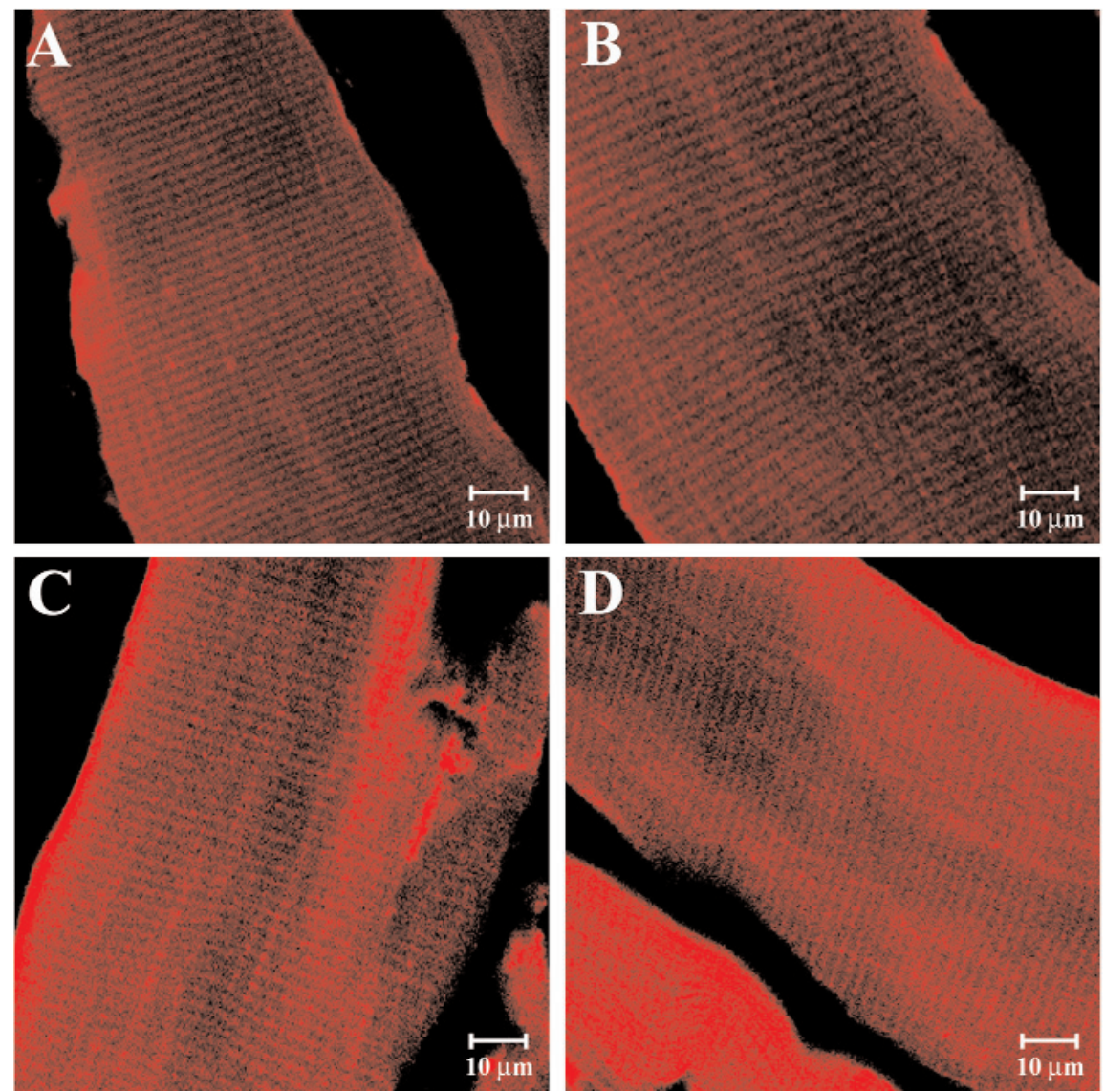

Fig. 3. Compound panel showing immunohistochemical findings, about integrins, in skeletal muscle fibers of masseter in nonalpha male chimpanzee. Skeletal muscle fibers were immunolabelled with antibodies against $\alpha 7 \mathrm{~A}-(\mathbf{A}), \beta 1 \mathrm{~A}-(\mathbf{B}), \alpha 7 \mathrm{~B}-(\mathbf{C})$, and $\beta 1 D$-integrin $(\mathbf{D})$. Immunostaining of $\alpha 7 \mathrm{~A}$ - and $\beta 1 \mathrm{~A}$-integrin was reduced in comparison to $\alpha 7 \mathrm{~B}$ - and $\beta 1 \mathrm{D}$-integrin.
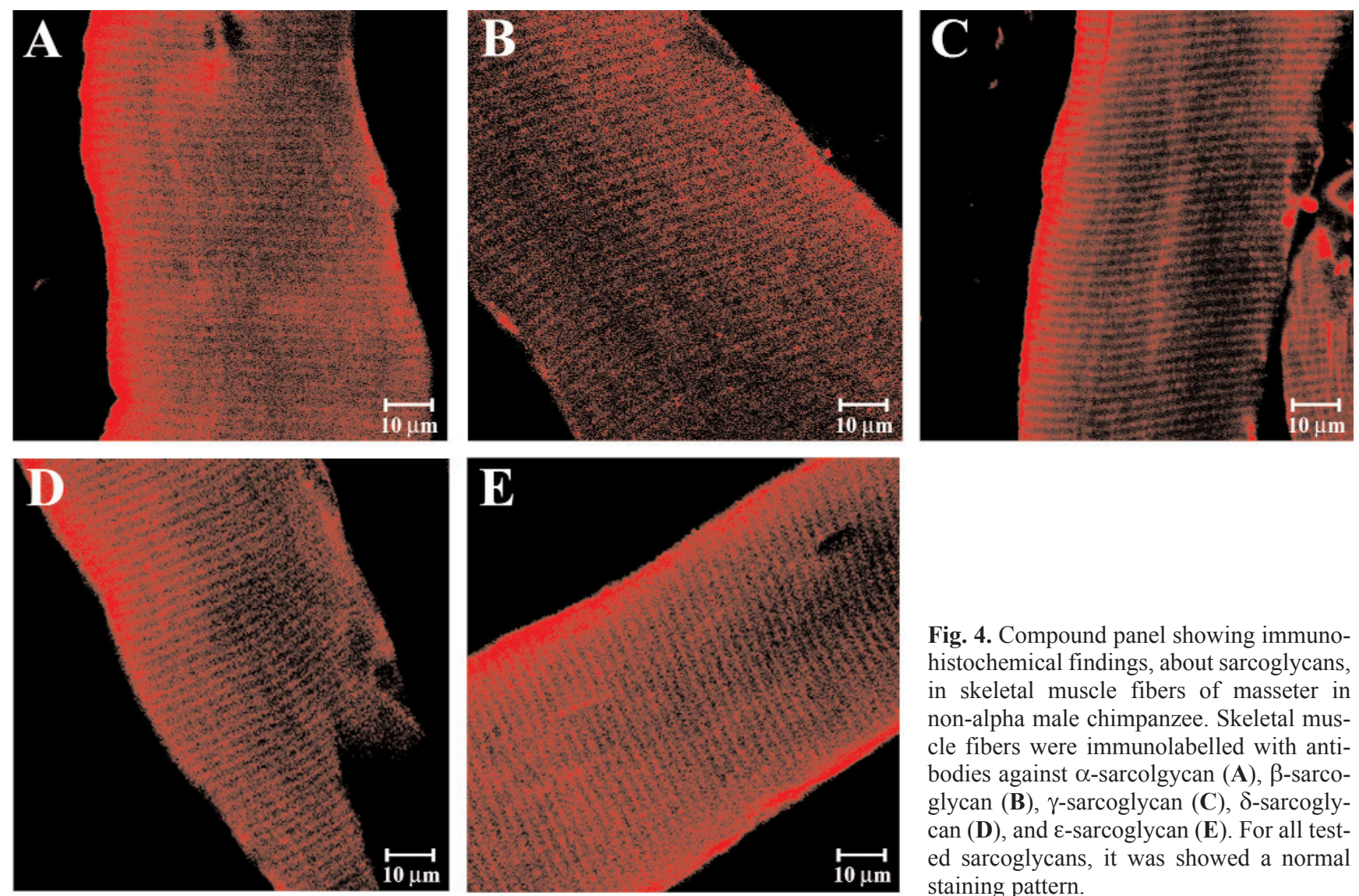

Fig. 4. Compound panel showing immunohistochemical findings, about sarcoglycans, in skeletal muscle fibers of masseter in non-alpha male chimpanzee. Skeletal muscle fibers were immunolabelled with antibodies against $\alpha$-sarcolgycan (A), $\beta$-sarcoglycan (B), $\gamma$-sarcoglycan (C), $\delta$-sarcoglycan (D), and $\varepsilon$-sarcoglycan (E). For all tested sarcoglycans, it was showed a normal staining pattern. 

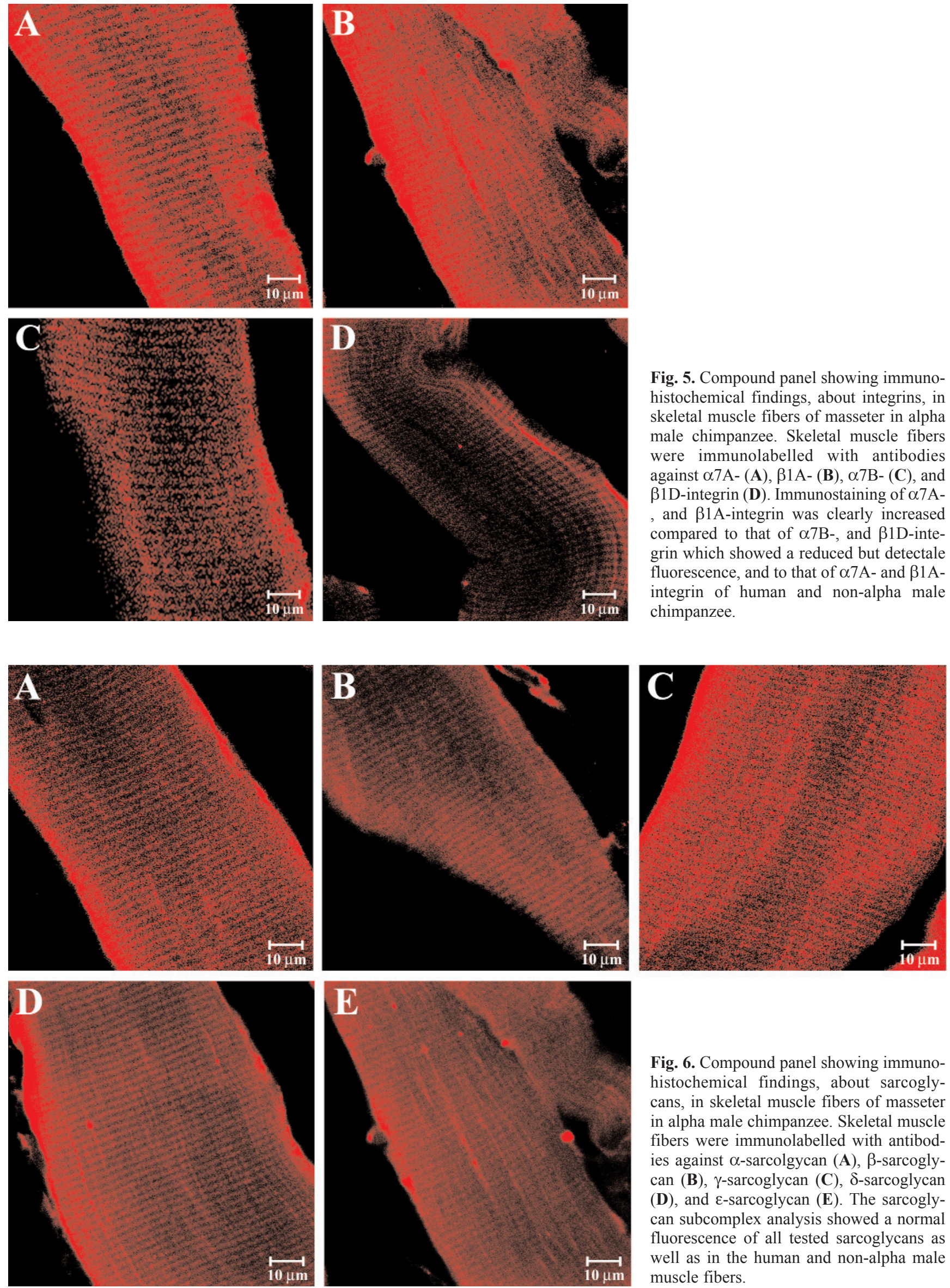

Fig. 6. Compound panel showing immunohistochemical findings, about sarcoglycans, in skeletal muscle fibers of masseter in alpha male chimpanzee. Skeletal muscle fibers were immunolabelled with antibodies against $\alpha$-sarcolgycan (A), $\beta$-sarcoglycan (B), $\gamma$-sarcoglycan $(\mathbf{C}), \delta$-sarcoglycan (D), and $\varepsilon$-sarcoglycan (E). The sarcoglycan subcomplex analysis showed a normal fluorescence of all tested sarcoglycans as well as in the human and non-alpha male muscle fibers.
Fig. 5. Compound panel showing immunohistochemical findings, about integrins, in skeletal muscle fibers of masseter in alpha were chimpanzee. Skeletal muscle fibers against $\alpha 7 \mathrm{~A}-(\mathbf{A}), \beta 1 \mathrm{~A}-(\mathbf{B}), \alpha 7 \mathrm{~B}-(\mathbf{C})$, and $\beta 1 D$-integrin (D). Immunostaining of $\alpha 7 \mathrm{~A}$ , and $\beta 1 \mathrm{~A}$-integrin was clearly increased . fluorescence, and to that of $\alpha 7 \mathrm{~A}$ - and $\beta 1 \mathrm{~A}$ integrin of human and non-alpha male chimpanzee. 
[34]. Thus, the progression from myoblast to myotubes is paralleled by increase in $\alpha 7 \mathrm{~A}$; this suggests that $\alpha 7$, isoform switching may be an important mechanism in skeletal muscle differentiation [35].

About isoform of $\beta 1$-integrin, it is showed that the $\beta 1 \mathrm{D}$-integrin chain is restricted to differentiated skeletal and cardiac muscle [36,37]; in contrast, the $\beta 1 \mathrm{~A}$ chain is present in a wide variety of cell types including myogenic precursor cells [22]. The $\beta 1 \mathrm{D}$ cytoplasmic domain may act to arrest the progression of myoblast proliferation and alter subcellular localization [20]; therefore, increased $\beta 1 \mathrm{D}$ expression may increase the interaction between the extracellular matrix, sarcolemma, and the cell cytoskeleton, stabilizing muscle integrity [22]. In this way, the shift from $\beta 1 \mathrm{~A}$ and increased $\beta 1 \mathrm{D}$-integrin reflects fewer mononuclear cell infiltrates and increased stability of muscle fibers [22].

Previously, it has been demonstrated that the loss of MYH16 gene, implied in jaw musculature regulation, in humans may have resulted in smaller masticatory muscle and consequential changes to craniofacial morphology with consequential expansion of the human brain case [10]. Besides, it was showed that protein expression in muscle fibers is highly plastic and it is unlikely that the inactivation of MYH16 would have associated with dramatic changes in hominin masticatory mechanics [38]. Based on this, powerful masticatory muscles are found in most primates, including chimpanzees and gorillas; in contrast, masticatory muscles are considerably smaller in both modern and fossil members of Homo [13].

On this basis, here, we hypothesized that the different quantitative composition of integrins, observed in muscle fibers of alpha-male, could be influenced by MYH16 gene. Then, in our opinion it is intriguing to hypothesize that the MYH16 gene, more expressed in chimpanzees and loss in human muscles, could modify the expression of integrins, influencing, in turn, the phenotype of muscle.

In this way, $\alpha 7 \mathrm{~A}-$ and $\beta 1 \mathrm{~A}$-integrin could determine the presence of type II fibers and then they could play a key role in the determination of contraction force. Then, MYH16 gene could be a common interactor of signalling between sarcoglycans and integrins in chimpanzee muscles.

This hypothesis, highlighting the influence of the gene expression by different social condition, as well as the different role of chimpanzee in the same group, open a new line of research in order to better comprehend the real mechanism on the basis of the evolution.

\footnotetext{
Aknowledgements: This work is supported by Regione Siciliana
} - Assessorato alla Sanita - "Fondo di Ricerca Sanitaria 2007".

\section{References}

[1] Huxley TH. Evidence as to man's place in nature. London: Williams and Norgate; 1863.

[2] Darwin C. The secret of man, and selection in relation to sex. New York: D Appleton and Company; 1871.

[3] McGrew WC. Chimpanzee material culture: implication for human evolution. Cambridge: Cambridge University Press; 1992.

[ 4] Donaldson I, Gottgens B. Evolution of candidate transcriptional regulatory motifs since the human-chimpanzee divergence. Genome Biol. 2006; 7: R52.

[5] Yunis JJ, Prakash O. The origin of man: a chromosomal pictorial legacy. Science. 1982;215: 1525-1530.

[6] Currat M, Excoffier L. Modern humans did not admix with Neanderthals during their range expansion into Europe. PLoS Biol. 2004;2:2264-2274.

[7] Wimmer R, Kirsch S, Rappold GA, Schempp W. Direct evidence for the Homo-Pan clade. Chromosome Res. 2002;10: 55-61.

[8] Dennehey BK, Gutches DG, McConkey EH, Krauter KS. Inversion, duplication, and changes in gene context are associated with human chromosome 18 evolution. Genomics. 2004;83: 493-501.

[9] Pollard KS, Salama SR, King B et al. Forces shaping the fastest evolving regions in the human genome. Plos Genet. 2006;2:1599-1611.

[10] Stedman HH, Kozyak BW, Nelson A et al. Nature. 2004;428: 415-418.

[11] Hohl TH. Masticatory muscle transposition in primates: effects on craniofacial growth. J Maxillofac Surg. 1983;11: 149-156.

[12] Brennan M, Antonyshyn O. The effects of temporalis muscle manipulation on skull growth: an experimental study. Plast Reconstr Surg. 1994;97:13-24.

[13] Rak Y. The Australopithecine Face. New York: Academic; 1983.

[14] Tobias PV. The skulls, endocasts, and teeth of Homo habilis. Cambridge: University Press; 1991.

[15] Clark EA, Brugge JS. Integrins and signaling transduction pathways: the road taken. Science (Wash DC). 1995;268:233239.

[16] Giancotti FG, Ruoslahti E. Integrin signaling. Science. 1999;285:1028-1032.

[17] Hynes RO. Integrins: versatility, modulation and signalling in cell adhesion. Cell. 1992;69:11-25.

[18] Juliano RL, Haskill S. Signaling transduction from the extracellular matrix. J Cell Biol. 1993;120:577-585.

[19] Sastry S, Horwitz A. Integrin cytoplasmic domains: mediators of cytoskeletal linkages and extra- and intracellular initiated transmembrane signaling. Curr Opin Cell Biol. 1993;5:819831.

[20] Belkin AM, Retta SF, Pletjushkina OY et al. Muscle 1D integrin reinforces the cytoskeleton-matrix link: modulation of integrin adhesive function by alternative splicing. $J$ Cell Biol. 1997:139:1583-1595.

[21] Belkin AM, Zhidkova NI, Balzac F et al. $\alpha 1 \mathrm{D}$ integrin displaces the $\beta 1 \mathrm{~A}$ isoform in striated muscle: localization at junctional structures and signaling potential in nonmuscle cells. J Cell Biol. 1996;132:211-226.

[22] Burkin DJ, Wallace GQ, Nicol KJ, Kaufman DJ, Kaufman SJ. Enhanced expression of the $\alpha 7 \beta 1$ integrin reduces muscular dystrophy and restores viability in dystrophic mice. $J$ Cell Biol. 2001;152:1207-1218.

[23] Anastasi G, Cutroneo G, Santoro G et al. Integrins, muscle agrin and sarcoglycans during muscular inactivity conditions: an immunohistochemical study. Eur J Histochem. 2006;50: 327-336 
[24] Anastasi G, Cutroneo G, Santoro G et al. Costameric proteins in human skeletal muscle during muscular inactivity. J Anat. 2008;213:284-295.

[25] Yoshida T, Pan Y, Hanada H, Iwata Y and Shigekawa M. Bidirectional signaling between sarcoglycans and the integrin adhesion system in cultured L6 myocytes. J Biol Chem. 1998; 273:1583-1590.

[26] Anastasi G, Cutroneo G, Trimarchi F et al. Sarcoglycans in human skeletal muscle and human cardiac muscle : a CLSM (Confocal Laser Scanning Microscope) study. Cells Tissues Organs. 2003a; 173:54-63.

[27] Anastasi G, Amato A, Tarone G et al. Distribution and localization of vinculin-talin-integrins system and dystrophin-glycoprotein complex in human skeletal muscle: immunohistochemical study using confocal laser scanning microscopy. Cells Tissues Organs. 2003b;175:151-164.

[28] Anastasi G, Cutroneo G, Rizzo G et al. Sarcoglycans and integrins behavior in normal human skeletal muscle: a CLSM (Confocal Laser Scanning Microscope) study. Eur J Histochem. 2004;48:245-252.

[29] Horwitz A, Duggan E, Buck C, Beckerle MC, Burridge K. Intyeraction of plasma membrane fibronectin receptor with talin-a transmembrane linkage. Nature (Lond.). 1986;320: 531-533.

[30] Otey CA, Pavalko FM, Burridge K. An interaction between actinin and the $\beta 1$ integrin subunit in vitro. J Cell Biol. 1990; 111:721-729.

[31] BenZeev A, Robinson GS, Bucher NRL, Farmer SR. Cellcell and cell-matrix interactions differentially regulate the expression of hepatic and cytoskeletal genes in primary cultures of hepatocytes. Proc Natl Acad Sci (USA). 1998: 85:2161-2165.

[32] Inberg DE and Folkman J. How does extracellular matrix control capillary morphogenesis? Cell. 1989;58:803-805.

[33] Ingber DE. Tensegrity: the architectural basis of cellular mechanotransduction. Annu Rev Physiol. 1997;59:575-599.

[34] Ziober BL, Vu MP, Waleh N, Crawford J, Lin CS, Kramer $\mathrm{RH}$. Alternative extracellular and cytoplasmic domains of the integrin alpha 7 subunit are differentially expressed during development. J Biol Chem. 1993;268:26773-26783.

[35] Hogervorst F, Kuikman I, Noteboom E, Sonnenberg A. The role of phosphorylation in activation of the alpha $6 \mathrm{~A}$ beta 1 laminin receptor. J Biol Chem. 1993;268:18427-18430.

[36] van der Flier A, Kuikman I, Baudoin C, van der Neut R, Sonnenberg A. A novel beta 1 integrin isoform produced by alternative splicing: unique expression in cardiac and skeletal muscle. FEBS Lett. 1995;369:340-344.

[37] Zhidkova NI, Belkin AM, Mayne R. Novel isoform of beta 1 integrin expressed in skeletal and cardiac muscle. Biochem Biophys Res Commun. 1995;214:279-285.

[38] McCollum MA, Sherwood CC, Vinyard CJ, Lovejoy CO, Schachat F. Of muscle-bound crania and human brain evolution: the story behind the MYH16 headlines. J Hum Evol. 2006;50:232-236

Submitted: 8 October, 2009 Accepted after reviews: 28 November, 2009 